\title{
The Role of Anatomical Location of Parotid Tumors in the Incidence of Post-Operative Facial Nerve Paresis
}

\author{
Hegab $\mathrm{AF}^{1 *}$ and Amer $\mathrm{Y}^{2}$ \\ ${ }^{1}$ Department of Oral \& Maxillofacial Surgery, Al-Azhar \\ University-Cairo, Egypt \\ ${ }^{2}$ Department of General surgery, Al-Azhar University- \\ Cairo, Egypt \\ *Corresponding author: Hegab AF, Department of \\ Oral \& Maxillofacial Surgery, Al-Azhar University in \\ Cairo, Egypt
}

Received: J une 16, 2017; Accepted: July 13, 2017; Published: July 20, 2017

\begin{abstract}
Objective: The parotid part of the Facial nerve dividing the parotid gland into the superficial and deep lobe. This close relation of the facial nerve to the parotid gland making the facial nerve at high risk of injury during parotid gland surgery. The current study aimed to evaluate the diagnostic accuracy of CT for evaluation of the anatomical location of parotid tumors and its relation to the incidence of post-operative facial nerve paresis with antegrade dissection technique.
\end{abstract}

Methods: The study enrolled the patients undergoing surgical removal of parotid tumors. Preoperative evaluation of the tumor location within the parotid gland tumor was done using axial and coronal CT. The primary outcome factor was the tumor location within the parotid gland. While secondary outcome factors were patient age, tumor size. Results: Deep lobes, upper and anterior part tumors are associated with higher incidence of the TFND $(P<0.001$, $<0.0001,<0.0349$, respectively). While, tumors located in the lower parts, posterior parts or the superficial lobe are showing lower incidence of TFND. With respect to tumor size, there was no significant association between the tumor size and incidence of facial nerve injury. The other clinical factors (age \& sex) did not show a relation to the incidence of the TFND.

Conclusion: The tumor location is the most significance factor of postoperative TFND. The incidence of TFND is highly significant with tumors located in the upper parts, and/or anterior parts and/or deep lobe of the parotid gland.

Keywords: Parotidectomy; Facial nerve morbidity; Antegrade dissection technique

\section{Introduction}

Surgical removal of the parotid tumor (Parotidectomy) was performed for the first time in 1832. Berard who removed parotid tumor of 8 years duration did the surgical procedure and introduced it into the world. Since then, a variety of modification has been proposed for treatment of benign and malignant parotid tumors [1].

Parotidectomy associated with a variety of postoperative complications including, Transient Facial Nerve Dysfunction (TFND), enduring facial nerve paralysis, salivary fistula, Frey's syndrome, infection, and recurrence of the parotid tumor. Due to location of the parotid gland in the facial region, complications of the parotid gland surgery lead to disfigurement and can affect the quality of life [2].

Owing to the anatomical relation between the facial nerve and the parotid gland; the incidence of facial nerve injury is the most frequent complication after Parotidectomy. In the same time; facial never identification in the diagnostic image is extremely difficult. That is giving us two options. The first is direct identification of the nerve during surgery, which carry a risk of injury to the nerve. The second option is to use anatomical landmark in the imaging study to predict the location of the nerve to the tumor.

Facial nerve injury associated with Parotidectomy can results from different mechanisms include nerve detachment or cutting, stretching, nerve compression from heavy retraction and suture ligation, ligature entrapment, thermal and electrical injuries, and finally can result from ischemia [3]. Facial nerve trunk identification during Parotidectomy can be achieved by one of the following approaches, conventional antegrade dissection [4] of the facial nerve, and retrograde dissection [5].

Some prefer to identify the facial nerve trunk first and continue dissection forward through the course of the facial nerve branches which called the antegrade approach. The nerve trunk may prove difficult find in obese patients, those with large benign tumors or during procedures for recurrent pleomorphic adenomas where retrograde dissection of the nerve branches at the periphery may provide a useful alternative [6]. A number of publications have renewed focus on the retrograde technique [7-10].

Different factors can lead to facial nerve injury (either temporary or permanent) during Parotidectomy. The effect of type of the tumor (benign or malignant), the tumor size, age and sex are well-studied [11-13].

The purpose of the current study was to use different anatomical landmarks on the diagnostic image to predict the tumor location in relation to the facial nerve. Moreover, we study the possible predictive 


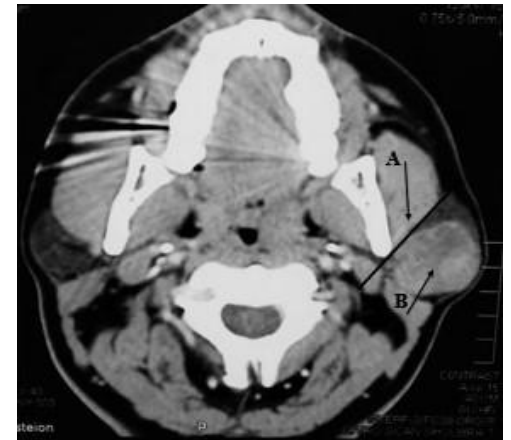

Figure 1: Facial Nerve (FN) line connects the lateral surface of the posterio belly of the digastrics muscle with the lateral surface of the cortex of the ascending mandibular ramus. A: the posterior belly of the digastric muscle. B: parotid tumor mass

clinical and anatomical factors related to the occurrence of peripheral facial paralysis with antegrade dissection technique.

\section{Patients and Methods}

In order to explore the factors that play a role in the incidence of facial nerve injury following Parotidectomy, this study recruited patients who had been diagnosed with a parotid diseases that mandated a surgical intervention. The study enrolled the patients of the outpatient clinics of the department oral and maxillofacial surgery- faculty of dental medicine, department of general surgery faculty of medicine, Al-Azhar University hospitals in Cairo, Egypt, between the years 2014 and 2016. This study was approved by IRB and all participants signed an informed consent agreement according to the ethics of clinical research committee. After obtaining written patient consent, appropriate surgical procedures for the treatment of parotid tumors were performed. Type of the Parotidectomy was selected after careful clinical and radiographic evaluation to determine the nature of the parotid tumor and location.

Inclusion criteria: patients with parotid gland tumors required superficial Parotidectomy or total Parotidectomy. Patient with recurrent parotid tumor but without history of previous nerve palsy.

Patients fulfilling one or more of the following criteria were excluded from the study: patients with collagen diseases, history of facial nerve palsy, neuromuscular disorders affecting the face, diabetic neuropathy. The follow up period extended to 8 months for the cases with facial nerve palsy. And patients still under follow up for the evaluation of the recurrence of the parotid gland tumors.

The clinical examinations include examination of facial nerve function before surgery. The radiological investigations includes one or more of the following: neck Ultra Sound (US): as primary screening to detect whether the enlargement is superficial or deep lobe swelling, solid or cystic, and well defined or ill defined, and to detect enlarged Lymph Node (LNs). Fine needle aspiration biopsy was used to differentiate benign lesions from malignant one. Detailed evaluation of the tumor location within the parotid gland was done with CT. Moreover, CT was used for evaluation of the cervical LN and suspected metastasis.

Evaluation of the location of the tumor within the parotid gland was done by using three anatomical reference lines on the CT. The

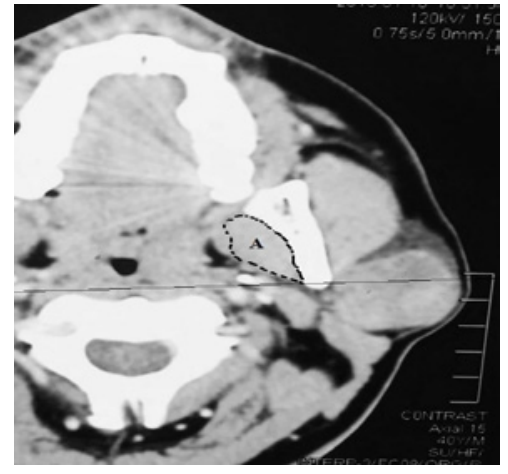

Figure 2: The coronal line at the posterior edge of the medial pterygoid muscle was defined as the A/P line in axial CT images. If the tumor was located behind the A/P line in axial CT images, the tumor was judged as being located in the posterior part of the parotid gland. If a part of the tumor reached the A/P line, the tumor was judged as being located in the anterior part of the parotid gland. A: the medial pterygoid muscle.

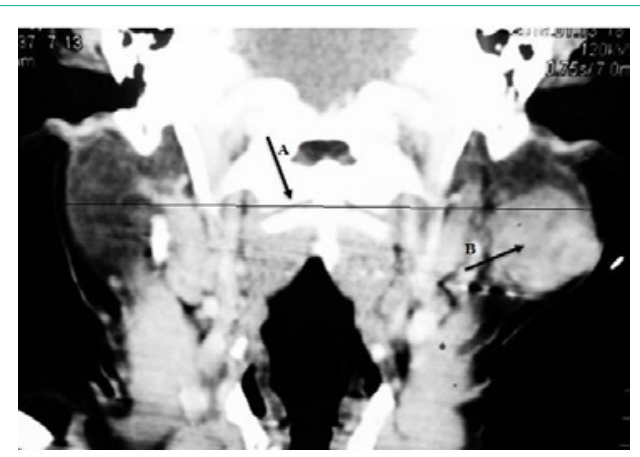

Figure 3: The horizontal line at the base of the odontoid process of the axis was defined as the $\mathrm{u} / \mathrm{l}$ line on coronal CT images. If the maximum width of the tumor was above the $\mathrm{U} / \mathrm{L}$ line in coronal CT images, the tumor was judged as being located in the upper part of the parotid gland; otherwise, the tumors were considered to be located in the lower. A: the base of the odontoid process of the axis. B: parotid tumor mass.

first line is the FN line: this line used for to determine whether the tumor was located in superficial or deep lobe; Facial Nerve (FN) line connects the lateral surface of the posterior belly of the digastrics muscle with the lateral surface of the cortex of the ascending mandibular ramus (Figure 1). If the tumor located anterior to this line will be considered as superficial lobe tumor. While tumor located behind the line will be considered as deep lobe tumor $[14,15]$.

The second line was the A/P line (anterior/posterior line) which created on the axial view of the preoperative CT [16]. The used for evaluation of the antero/posterior location of the tumor within the parotid gland. The medial pterygoid muscle is the keystone in this method. After complete outline of the medial pterygoid muscle, a horizontal line (coronal line) passing through the posterior edge of the medial pterygoid muscle in then axial CT. the tumor located anterior to that line will be considered as tumor of the anterior part of the parotid gland. Whereas the tumor cross that line or located behind the line will be considered as tumor in the posterior part of the parotid gland (Figure 2).

The third line is the U/Lline, which used for evaluation of the upper or lower location of the tumor within the parotid gland in the coronal CT. in this method, the odontoid process was used to 
evaluate the location of the tumor. In the coronal view of the CT, a horizontal line pass through the base of the odontoid process of the axis was created. If the maximum width of the tumor or all the tumor bulk was below that line, then the tumor will be considered as tumor at the lower part of the parotid gland. If the tumor is located above that line, the tumors were considered to be located in the upper part of the parotid gland (Figure 3 ).

The relation of the tumor to the branches of the facial nerve and location of the tumor within the superficial lobe or deep lope of the gland was assessed intraoperative by direct visualization. The intraoperative results were reported and correlated with the results obtained from the CT.

During operative procedures; if the center of the tumor is located at a level lower than the marginal mandibular branch, then tumor will be defined as lower pole tumors.

If the tumor in the parotid gland was benign or malignant located in the superficial lobe lateral to the facial nerve and not involving it, then superficial Parotidectomy will be performed.

While, total conservative Parotidectomy will be performed in the following cases: Resection of benign salivary tumors occupying both deep and superficial lobes of the parotid gland and low-grade malignant tumors not involving the facial nerve.

\section{Surgical technique}

The same surgical team performed all of the operations. Access to the parotid gland was done using the modified Blair incision. Antegrade dissection is performed in all the patients enrolled in the current study. The tragal pointer method is used for the identification of the facial nerve trunk. The dissection proceeds toward the peripheral branches with concurrent mobilization of parotid tissue anteriorly and laterally.

The flap dissection was kept in the proper subcutaneous plane outside the parotid capsule, with careful dissection when the terminal branches were reached. Skin hooks were used to apply vertical traction. To reduce the risk of traction injury, tissue was spread perpendicular to the incision and thus parallel to the direction of the main trunk of the nerve. If the tumor was in contact with the facial nerve, the facial nerve was dissected carefully from the tumor capsule. In deep tumor, the part of facial nerve in contact with the tumor was fully dissected and peeled off. The tumor was then removed from the bottom of the facial nerve. Vertically oriented blunt dissection minimizes the risk of injury to the distal branches of the facial nerve. Once the nerve trunk was identified we did not use diathermy at all; hemostasis was performed with surgical ligatures. For parenchymal division, we divided the substance of the parotid gland sharply and used ligatures as appropriate when bleeding was encountered. Gentle retraction and fine curved artery forceps were applied. The artery forceps were placed immediately above the nerve and then opened to carefully divide the bridging tissue over the nerve. Heavy pressure should not be applied on the dissected facial nerve by way of a dry swab or an excessively hot pack in the interest of hemostasis. Saline irrigation of the dissection field was carried out as the nerve dissection advanced. For total conservative Parotidectomy, once mobilization was completed we placed fine vascular slings beneath the nerve and very gently lifted it away from the tumor and continued dissection.

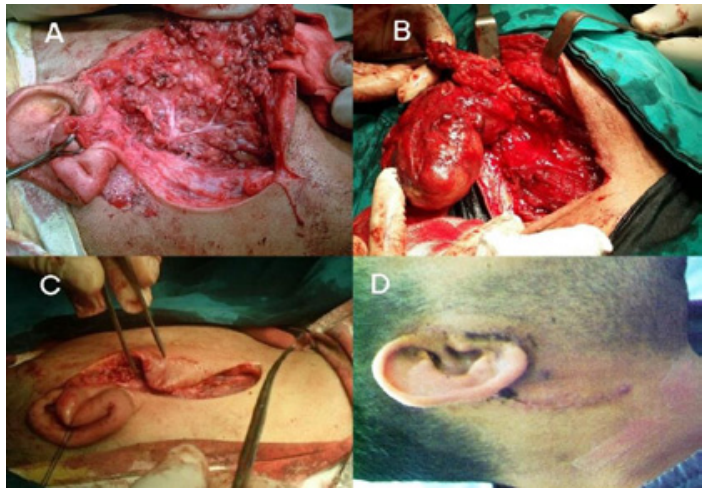

Figure 4: A modified Blair incision is used for access to the parotid gland. Ante grade dissection is performed by identifying the facial nerve trunk using the tragal pointer method. After initial identification of the nerve trunk, dissection proceeds toward the peripheral branches with simultaneous mobilization of parotid tissue anteriorly and laterally.

After surgical removal of the parotid tumor, suction drain was inserted and the wound was closed in two layers (subcutaneously with vicryl $3 / 0$ and proline 5/0 for skin). The drain removed after $24-48$ hours and the sutures were removed on the 5-7 days postoperatively (Figure 4).

\section{Postoperative management}

A pressure bandage was applied immediately after the operation. This was changed daily over the first few days. Antibiotic therapy was given in selected cases (e.g.; diabetic patients). Patients were asked about the presence of pain and the wounds were daily. The assessment was done clinically in the outpatients' clinic. In case of postoperative swelling, sonographic evaluation for the swelling will be done to differentiate the nature of the swelling (fluid accumulation or hematoma). Then after; the fluid accumulations were drained locally, and local antiseptic therapy and systemic antibiotic therapy was given if necessary. After complete resolution of the fluid accumulations, the function of the facial nerve was once again assessed clinically using the House-Brackmann scale. The patients associated with facial nerve dysfunction were followed up until complete facial never recovery observed. The facial nerve branches and the degree of the nerve injury were evaluated and reported in every visit. The time for facial nerve recovery and associated degree of TFND was evaluated, reported and statistically evaluated.

The House-Brackmann facial nerve injury grading system was used to grade facial nerve recovery following the Parotidectomy (Table 1) [17].

\section{Statistical analysis}

The primary variables were the Extent of surgery, histopathological findings, tumor size, and tumor depth. The secondary variables were the sex and age of the patient. These variables were studied in a chi 2 statistical univariate and stratified analysis to determine their association with postoperative facial nerve dysfunction.

Occurrences of TFND were studied in terms of the following clinical factors: patient's age, tumor size, histopathological diagnosis, and the location of the tumor within the parotid gland (superficial or deep lobe; upper or lower part; and anterior or posterior part). 
Table 1: House-Brackmann facial nerve grading system.

\begin{tabular}{|c|c|c|}
\hline Grade & Description & Characteristic \\
\hline Grade I & Normal & Normal facial function in all areas \\
\hline Grade II & Slight Dysfunction & $\begin{array}{l}\text { Gross: slight weakness noticeable on close inspection } \\
\text { At rest: normal symmetry and tone } \\
\text { Motion: forehead - moderate to good function; eye - complete closure with minimum effort; mouth - slight asymmetry. }\end{array}$ \\
\hline Grade III & Moderate Dysfunction & $\begin{array}{l}\text { Gross: obvious but not disfiguring difference between two sides } \\
\text { At rest: normal symmetry and tone } \\
\text { Motion: forehead - slight to moderate movement; eye - complete closure with effort; mouth - slightly weak with } \\
\text { maximum effort. }\end{array}$ \\
\hline Grade VI & Moderate Severe Dysfunction & $\begin{array}{c}\text { Gross: obvious weakness and/or disfiguring asymmetry } \\
\text { At rest: normal symmetry and tone } \\
\text { Motion: forehead - none; eye - incomplete closure; mouth - asymmetric with maximum effort. }\end{array}$ \\
\hline Grade V & Severe Dysfunction & $\begin{array}{c}\text { Gross: only barely perceptible motion } \\
\text { At rest: asymmetry } \\
\text { Motion: forehead - none; eye - incomplete closure; mouth - slight movement }\end{array}$ \\
\hline
\end{tabular}

The data were analyzed using Instat (GraphPad, CA, USA) statistical software. Mean (SD), medians, and percentages were calculated for the summarized data. The Wilcoxon rank sum test was used to assess the significance of differences among the groups. Probabilities of $<0.05$ were accepted as significant.

The sample size calculation was performed using $\mathrm{G}^{\star}$ Power version 3.1.9.2. The power was found to be 0.97 (97\%), indicating that the sample size was adequate.

\section{Results}

The final sample of the current study included 52 consecutive patients ( 31 males and 21 females) who underwent surgery for the treatment of parotid tumors. The patient age range from 33years to 67 years (mean 47.1, SD + 9.1). Incidence of postoperative facial nerve dysfunction was 33\% (17 patients) on the first postoperative day. The incidence of facial nerve injury was statistically significant of the cases when the tumor was in close relation to the facial nerve. Inflammatory conditions associated with superficial Parotidectomy, play an important role in the incidence of postoperative facial nerve dysfunction.

House-Brackmann grade II facial dysfunction was occurred in twelve patients out of the total patients with TFND (70\%). While grade III represent $24 \%$ (4 patients) and grade IV represent $1 \%$ (one patient). In our study; no patient developed complete facial nerve dysfunction on the first day after the operation. Complete facial nerve recovery occurred in all the affected patients. The median period of recovery was 2.6 months.

Marginal mandibular branch was the most affected branch with TFND (13 patents out of 17, 76\%). Followed by the frontal-Zygomatic branches (8 patients out of $17,47 \%$ ).

27 cases out of 52 (52\%) had Pleomorphic adenomas. And TFND occurred in only 7 cases $(26 \%$ of the total cases of Pleomorphic adenomas). While Warthin's tumor represents $31 \%$ of the total cases enrolled in the study (16 patients) and associated with 13\% of TFND (2 patients). The occurrence of TFND in pleomorphic adenoma was significant higher than with Warthin's tumor $(\mathrm{P}<0.05)$.

Mucoepidermoid carcinoma, Undifferentiated carcinoma, and adenocarcinoma occurred in 9 cases and 8 cases showed TFND (89\%). Only one case of undifferentiated carcinoma in the superficial lobe of the parotid gland was completely free form the facial nerve injury (Figure 5).

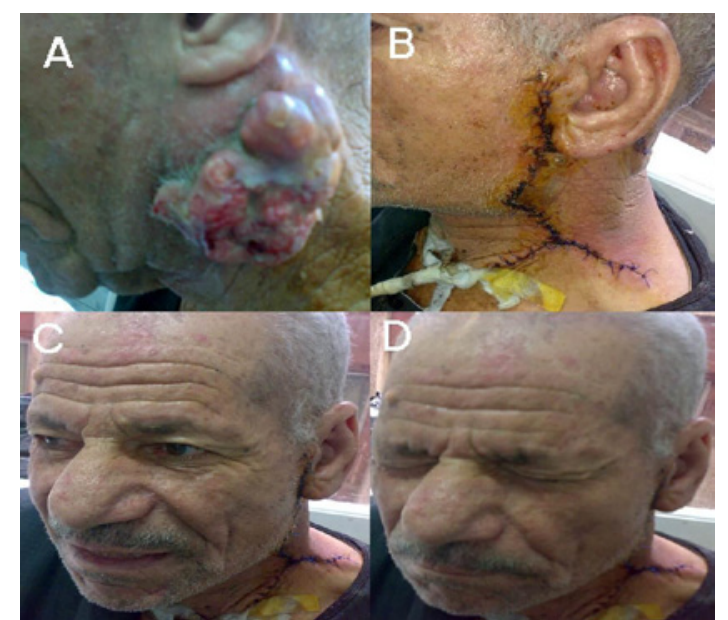

Figure 5: Case of undifferentiated carcinoma in the superficial lobe of the parotid gland was completely free form the facial nerve injury.

With respect to tumor size, there was no significant association between the tumor size and incidence of facial nerve injury.

In relation to the tumor depth, 19 tumors located in the deep part while 33 tumors were located in the superficial part of the gland. The incidence of the TFND was significant high in cases of tumors located in the deep part of the parotid gland $(\mathrm{P}<0.0001)$. In terms of; 14 cases of the facial nerve injury were located in the deep part of the gland while only three cases were located in the superficial part.

In the current study, upper part tumors were present in 18 patients while lower part tumor was present in 34 cases. Statistical analysis of the incidence of TFND showed significant incidence of TFND in cases of upper part tumors (12/18 associated with TFND, $67 \%)$ while the incidence was low in cases of lower part tumors $(5 / 34$ associated with TFND, 15\%). Statistical analysis of the incidence of TFND between upper and lower part tumors showed highly significant difference $(\mathrm{P}<0.0001)$.

Statistical analysis of the incidence of TFND between the anterior part and posterior parts tumors showed significant difference ( $\mathrm{P}$ $=0.0359$ ). In terms of; TFND occurred more in tumors located in the anterior part (10 of the 20 tumors located in the anterior part of the gland, 50\%) than in tumors located in the posterior part (7 of the 32 tumors, 22\%). The other clinical factors (age \& sex) did not show a relation to the incidence of the TFND. 
With regard to the extent of the surgery; the durations of surgery range from 70 to 150 minutes (mean of 95 minutes). The likelihood of an injury was higher with increase of the surgical time. In terms of; Surgeries that resulted in facial nerve injury had significantly higher surgical durations time than those not resulting in facial nerve injury $(\mathrm{P}<0.001)$.

\section{Discussion}

The close anatomical relation of the facial nerve and parotid gland lead to high incidence of TFND with parotid surgery. It's believed that the facial never course within the parotid gland divided it into superficial and deep lobe. This concept is not correct anatomically based. The course of the facial nerve within the parotid gland is passing through a potential plane, which lies in between the deep and superficial lobes of the parotid gland. The surgeon used the facial nerve course to identify that plane during surgical dissection [18].

The incidence of TFND with Parotidectomy ranged from $10 \%$ to $50 \%$. The cause of temporary weakness is neuropraxia. Neurapraxia of the facial nerve can be happened as a result of different form of trauma to the facial nerve during surgery. Different factors attributed to the incidence of TFND with Parotidectomy. The factors include dissection trauma to the facial nerve, electro-cautery thermal injury to the facial nerve, and increase the operating time. Permanent facial nerve injury occurred because of transaction or cautery to the main nerve trunk and its reported incidence is $0.5 \%[19,20]$.

The incidence of postoperative TFND differs according to the type of pathology and the length of surgery. Superficial Parotidectomy associated with $16.5 \%$ TFND for benign tumors, while malignant tumors associated with 13\% TFND. Whereas, total Parotidectomy increased the incidence of TFND to $31 \%$ for benign and $100 \%$ for malignant tumors in the early post-operative period [13].

Facial nerve injury may also be caused by accidental trauma during suture ligation, mainly during wound closure. To avoid this unnecessary complication, careful suturing under direct visualization should be performed.. Also, the use of electro-cautery in deep sites that are potentially close to facial nerve branches, or within the parotid gland, should also be avoided or minimized. Besides, in an attempted to stop bleeding during surging, good lightening and suction should be applied all the time to provide good visualization of the facial nerve branches. Otherwise; crushing or clamping tissue extensively in an attempt to stop bleeding and the heavy handed retraction of the tissue can result in direct trauma to the facial nerve branches [21].

Retrograde dissection technique is mostly used during revision Parotidectomy. While, the Anterograde dissection technique is meant to identifying the facial nerve at its point of exit from the stylomastoid foramen and is the preferred method of dissecting the facial nerve.

In the current study; the incidence of TFND was highly significant in cases of tumor located in the upper part of the parotid gland in comparison to tumor located in the lower part of the parotid gland $(\mathrm{P}<0.0001)$. This can be explained by; the upper part of the parotid gland is thin and tumor located in that part will be so close to the facial nerve branches. So, during surgical removal of the tumor from the upper part, excessive manipulation of the facial nerve branches is expected. This excessive manipulation can result in direct and indirect trauma to the facial nerve which results in TFND [22,23].

Second, tumor located in the anterior part of the parotid gland was associated with significant incidence of TFND in comparison to tumor located in the posterior part of the parotid gland ( $\mathrm{P}=0.0359$ ). This can be clarified by, to remove tumor from the anterior part of the parotid gland, we need to dissect a longer section of the facial nerve branches. Dissection of longer section of the facial nerve branches required more manipulation, stretching and retraction which increase the risk of TFND $[22,24]$.

Finally, the incidence of TFND was highly significant in cases of tumor located in the deep part of the gland $(\mathrm{P}<0.0001)$. This can be explained by, during surgical removal of tumor from the deep part of the parotid gland, more number of facial nerve branches included in the surgical field. Beside, deep tumors required more dissection and retraction of the tissue for longer time. All of the previously mentioned causes lead to increase trauma to the facial nerve with increase the incidence of the nerve injury.

With regards to the tumor size; there was non-significant correlation between the incidence of the TFND and tumor size. Even the tumor is small but located in the deep part in the gland, the incidence of the TFND is high due to the deep location of the tumor within the gland not because of the size. While; bigger superficial tumor is associated with lower incidence of TFND due to superficial location of the tumor rather than the tumor size. So, the tumor location within the parotid gland is the most important factor related to the incidence of the TFND.

With regard to the incidence of the TFND per branch, the higher i9ncidenec occurred with the Marginal mandibular branch (13 patents out of $17,76 \%$ ). The second most frequent incidence of the TFND associated with the frontal-Zygomatic branches (8 patients out of 17, 47\%). Increase of the incidence of TFND with Marginal mandibular branch injury could be owing increase the manipulation and handling of the lower branches of facial nerve during operation. The result of the current study in agreement with the results previously reported studies showing higher incidence of the TFND with the marginal mandibular branch [12,25-26].

One of the limitations of our study is small number of the patients. Studies with larger number of patients or systematic review are recommended to evaluate the incidence of TNFD per facial nerve branch.

With regards to the effect of the duration of the surgery ion the incidence of TFND; in the current study, increase the surgical time associated with significantly higher of TFND. The longer duration of surgery resulting in longer time of retraction and excessive swelling formation, which could cause pressure on the facial nerve. This pressure can result in indirect trauma to the facial nerve with subsequent TFND.

The other factors, including patient's age, sex, and tumor size, were not apparent risks for TFND.

\section{Conclusion}

Parotidectomy is a technically demanding operation. The tumor location is the most significance factor of postoperative TFND. The 
incidence of TFND is highly significant with tumors located in the upper parts, and/or anterior parts and/or deep lobe of the parotid gland.

\section{References}

1. Wormald R, Donnelly M, Timon C. 'Minor' morbidity after parotid surgery via the modified Blair incision. J Plast Reconstr Aesthet Surg. 2008; 62: 10081011.

2. Henney SE, Brown R, Phillips DE. Parotidectomy: the timing of postoperative complications. Eur Arch Otorhinolaryngol. 2010; 267: 131-135.

3. Eisele DW, Wang SJ, Orloff LA. Electrophysiologic facial nerve monitoring during parotidectomy. Head Neck. 2010; 32: 399-405.

4. Snow G. The surgical approaches to the treatment of parotid pleomorphic adenomas Controversies in the Management of Salivary Gland Disease: Oxford University Press. 2001; 57-66.

5. Chan S, Gunn A. Conservative parotidectomy by the peripheral approach. Br J Surg. 1981; 68: 405-407.

6. McGurk M, Williams RG, Calman F. A clinical approach to malignant tumours Colour Atlas and Text of Salivary Glands: Diseases, Disorders and Surgery London: Mosby-Wolf. 1995; 181-196.

7. O'Regan B, Bharadwaj G, Bhopal S, Cook V. Facial nerve morbidity after retrograde nerve dissection in parotid surgery for benign disease: A 10-yea prospective observational study of 136 cases. $\mathrm{Br} \mathrm{J}$ Oral Maxillofac Surg. 2007; 45: 101-107.

8. Bhattacharyya N, Richardson ME, Gugino LD. An objective assessment of the advantages of retrograde parotidectomy. Otolaryngol Head Neck Surg. 2004; 131: 392-396.

9. Wang DZ, Liu SJ, Donoff RB, Guralnick W. A modified centripetal approach to parotidectomy. J Oral Maxillofac Surg. 1985; 43: 14-19.

10. Yu GY. Superficial parotidectomy through retrograde facialnerve dissection. J R Coll Surg Edinb. 2001; 46: 104-107.

11. Laccourreye $\mathrm{H}$, Laccourreye $\mathrm{O}$, Cauchois $\mathrm{R}$, Jouffre $\mathrm{V}$, Me nard M, Brasnu $\mathrm{D}$. Total conservative parotidectomy for primary benign pleomorphic adenoma of the parotid gland: a 25-year experience with 229 patients. Laryngoscope. 1994; 104: 1487-1494.

12. Mra Z, Komisar A, Blaugrund SM. Functional facial nerve weakness after surgery for benign parotid tumors: a multivariate statistical analysis. Head Neck. 1993; 15: 147-152.
13. Bron LP, O'Brien CJ. Facial nerve function after parotidectomy. Arch Otolaryngol Head Neck Surg. 1997; 123: 1091-1096.

14. Lim CY, Chang HS, Nam KH, Chung WY, Park CS. Preoperative prediction of the location of parotid gland tumors using anatomical landmarks. World J Surg. 2008; 32: 2200-2203

15. Conn IG, Wiesenfeld D, Ferguson MM. The anatomy of the facial nerve in relation to CT/sialography of the parotid gland. $\mathrm{Br} \mathrm{J}$ Radiol. 1983; 56: 901 905.

16. Ikoma R, Ishitoya J, Sakuma Y, Hirama M, Shiono O, Komatsu M, et al. Temporary facial nerve dysfunction after parotidectomy correlates with tumor location. Auris Nasus Larynx. 2014; 41: 479-484.

17. House TW, Brackmann DE. Facial nerve grading system. Otolaryngol Head Neck Surg. 1985; 93: 146-147.

18. Saha S, Pal S, Sengupta M, Chowdhury K, Saha VP, Mondal L. Identification of Facial Nerve During Parotidectomy: A Combined Anatomical \& Surgical Study. Indian J Otolaryngol Head Neck Surg. 2014; 66: 63-68.

19. O'Brien CJ. Current management of benign parotid tumors-the role of limited superficial parotidectomy. Head Neck. 2003; 25: 946-952.

20. Leverstein $\mathrm{H}$, van der Wal JE, Tiwari RM et al. Surgical management of 246 previously untreated pleomorphic adenomas of the parotid gland. $\mathrm{Br} \mathrm{J}$ Surg. 1997; 84: 399-403

21. Elmadawy A, Hegab A, Alahmady H, Shuman M. Clinical and electromyographic assessment of facial nerve function after temporomandibular joint surgery, Int J Oral Maxillofac Surg. 2015; 44: 1275-1280.

22. Gaillard C, Périé S, Susini B, St Guily J. Facial nerve dysfunction after parotidectomy: the role of local factors. Laryngoscope. 2005; 115: 287-291.

23. Dulguerov $P$, Marchal F, Lehmann W. Postparotidectomy facial nerve paralysis: possible etiologic factors and results with routine facial nerve monitoring. Laryngoscope. 1999; 109: 754-762.

24. Cannon CR, Replogle WH, Schenk MP. Facial nerve in parotidectomy: a topographical analysis. Laryngoscope. 2004; 114: 2034-2037.

25. Nichols RD, Stine PH, Bartschi LR. Facial nerve functions in 100 consecutive parotidectomies. Laryngoscope. 1979; 89: 1930-1934.

26. Davis RA, Anson BJ, Budinger JM, Kurth LR. Surgicalanatomy of the facia nerve and parotid gland basedupon a study of 350 cervicofacial halves. Surg GynecolObstet. 1956; 102: 385-413.
Austin J Surg - Volume 4 Issue 2 - 2017 ISSN : 2381-9030 | www.austinpublishing group.com Hegab et al. (C) All rights are reserved
Citation: Hegab AF and Amer Y. The Role of Anatomical Location of Parotid Tumors in the Incidence of PostOperative Facial Nerve Paresis. Austin J Surg. 2017; 4(2): 1102. 